\title{
ANÁLISE DA GORDURA CORPORAL POR ANALISADORES E PESO HIDROSTÁTICO DE MULHERES MILITARES DO EXÉRCITO BRASILEIRO
}

\author{
Body fat analysis of Brazilian army women by hydrostatic weigh analyzers \\ Ana Beatriz Moreira de Carvalho Monteiro', Candido Simões Pires-Neto², \\ José Fernandes Filho ${ }^{3}$
}

\section{RESUMO}

Os valores da composição corporal em dois componentes, massas de gordura e corporal magra, de mulheres militares brasileiras, avaliadas pela pesagem hidrostática $(\mathrm{PH})$, em relação aos valores informados por distintos analisadores de impedância bioelétrica, são desconhecidos. Esse estudo objetivou comparar e correlacionar a gordura relativa (G\%), obtida pela técnica de pesagem hidrostática, com a G\% fornecida por analisadores de gordura corporal em mulheres militares do Exército Brasileiro (MMEB). A amostra foi composta de 30 MMEB, com idade média de $32,80 \pm 5,7$ anos, MC kg $=57,4 \pm 6,1 \mathrm{~kg}$, EST $\mathrm{cm}=164,8 \pm 5,8 \mathrm{~cm}$, densidade corporal $=1,044429 \pm 0,00946 \mathrm{~g} / \mathrm{cm}^{3}$ e G\% $=23,9 \pm 4,2 \%$. Foram correlacionados e comparados os valores da G\% obtidos pelos analisadores Maltron BF 900, Omron HBF 3000, Tanita Ultimate Scale 2001 e Futrex 5000 A/ZL com a G\% obtida via PH. Para a análise dos dados, usou-se a correlação de Pearson, e o teste t pareado de Student ( $p \leq 0,05$ ) entre a G\% critério e a informada por cada analisador, no SPSS v10. Houve significante correlação $(p<0,05)$ da $G \%$ determinada pelos analisadores Maltron $(r=0,69 ; p=0,001)$, Omron $(r=0,62 ; p=0,001)$, Tanita $(r=0,75$; $p=0,001)$ e Futrex 5000 ( $r=0,65 ; p=0,001)$ com a $G \%$ obtida pela $\mathrm{PH}$. Não houve diferença significativa entre a $\mathrm{G} \%$ $(p>0,05)$ de cada analisador, Maltron $(p=0,78)$, Omron $(p=0,49)$ e Futrex $5000(p=0,44)$ com a G\%, via PH, exceto para o Tanita, $p>0,05$. Concluindo, o Tanita foi o único analisador não adequado para estas militares.

Palavras-chave: Composição Corporal, Pesagem Hidrostática, Analisadores de Gordura Corporal.

\section{ABSTRACT}

The Brazilian Army females body fat (\%F) measured by hydrostatic weighing (HW) in relation to values from bioelectric impedance analysis (BIA) are completely unknown. Consequently, the present study aimed to compare and correlate body fat measured by hydrostatic weighing of Brazilian Army females (BAF) and by means of electrical devices such as Maltron BF 900, Omron HBF 3000, Tanita Ultimate Scale 2001 and Futrex 5000 A/ZL. The sample was formed by $30 \mathrm{BAF}$, with average age of $32.8 \pm 5.7 \mathrm{y}$, body mass $=57.4 \pm 6.1 \mathrm{~kg}$, height $=164.8 \pm 5.8$ $\mathrm{cm}$, body density by hydrostatic weighing = $1.044429 \pm 0.00946 \mathrm{~g} / \mathrm{cm}^{3}$ and $\% \mathrm{~F}=23.9 \pm 4.2 \%$. The $\% \mathrm{~F}$ measured by each analyzer was first correlated and thereafter compared to \%F from HW. Statistics consisted of descriptive, a dependend $t$ test between \%F from HW and \%F from each analyzer and as well as for Pearson correlation ( $p \leq 0.05$ ) were analyzed by SPSS v10. There was a significant correlation between \%F measured by means of Maltron $(r=0.69 ; p=0.001)$, Omron ( $r=0.62$; $p=0.001)$, Tanita $(r=0.75 ; p=0.001)$, Futrex $(r=0.65 ; p=0.001)$ and the one measured by HW. There were no significant differences between mean $\% F, p>0.05$, from HW compared to Maltron, $p=0.78$, Omron, $p=0.49$, and Futrex, $p=0.44$, except for Tanita, $p<0.05$. Therefore, the only inadequate device for these military women was the Tanita.

Key words: Body Composition, Hydrostatic Weighing, Body Fat Devices.

1. Universidade Estácio de Sá - Rio de Janeiro - RJ - Brasil.

2. UNIGUAÇU - União da Vitória - PR - Brasil.

3. Universidade Castelo Branco - Rio de Janeiro - RJ - Brasil.

Recebido em 10.06.2008. Aceito em 25.09.2008.

Revista de Educação Física 2008 Dez; 143:3-11. Rio de Janeiro (RJ) - Brasil. 


\section{INTRODUÇÃO}

Sabe-se que o uso de analisadores da impedância bioelétrica, $\mathrm{BIA}^{1}$, e da NIR, ou interactância quase-infravermelha ou espectrofotometria infravermelha ${ }^{2}$, além de serem técnicas não invasivas, não oferecem riscos ao avaliado, quando da determinação de sua gordura corporal relativa.

Resumidamente, a técnica de bioimpedância está fundamentada no trânsito de uma corrente elétrica de baixíssima intensidade, que passa pelo corpo $^{3}$, e fundamenta-se em três princípios: a resistência, $R$, que é a oposição natural que o corpo oferece ao trânsito da corrente elétrica; a reatância, $\mathrm{Xc}$, que é a oposição ao fluxo dessa corrente causada pela capacitância produzida pela membrana celular; e pela impedância (Z), ou seja, a raiz quadrada da soma dos quadrados da resistência e reatância ao fluxo daquela corrente elétrica medida pelo analisador. $O$ fluxo elétrico é facilitado, na massa corporal magra (MCM, kg), devido à sua quantidade de líquidos (água), que, normalmente, oscila entre 68 e $75 \%$ em adultos, além de por eletrólitos ${ }^{4}$. Como os tecidos adiposos e ósseos são péssimos condutores de eletricidade, devido aos seus baixíssimos conteúdos de água, uma pessoa com uma maior quantidade de massa muscular (e, também, de líquidos) que integra a MCM, oferecerá uma menor oposição ao trânsito da corrente elétrica emitida pelo analisador.

Por sua vez, a técnica da NIR baseia-se nos princípios de absorção e reflexão da luz, através da densidade ótica (DO), usando um espectrofotômetro infravermelho ${ }^{5}$. A luz refletida é convertida para valores de $\mathrm{DO}$ e, como o tecido adiposo absorve luz e a MCM, através de seus componentes (proteína, músculo), reflete luz, pode-se estimar a G\%, através de procedimentos matemáticos registrados no programa do equipamento. Valores menores de DO indicam uma maior absorção da luz infravermelha que, por sua vez, reflete uma maior quantidade de gordura no local de avaliação, refletindo, em termos de interpretação, a gordura corporal relativa.

Relatos de Lukaski ${ }^{6}$, Heyward et al. ${ }^{7}$ e Lohman ${ }^{8}$ colaboraram, enormemente, para o desenvolvimento de procedimentos nacionais para o uso de analisadores da G\% ${ }^{9-14}$.

Distintos autores, como Cable et al. ${ }^{15}$, Eaton et al. ${ }^{16}$, Eckerson et al. ${ }^{17}$, Heyward ${ }^{1}$, Smith et al. ${ }^{18}$ e Utter et al. ${ }^{19}$, compararam e correlacionaram os valores da G\% obtidos pelas técnicas antropométricas, da BIA e NIR, com aqueles obtidos via pesagem hidrostática $(\mathrm{PH})$, em homens e mulheres. Mas, como estes estudos diferem em relação às atividades, funções e características antropométricas dos avaliados, todos não militares, torna-se importante desenvolver estudos que analisem, em um mesmo grupo de mulheres militares, o uso de distintas técnicas para a determinação da G\%. Ao que se sabe, esse é o primeiro artigo nacional que analisa, simultaneamente, a gordura relativa corporal de mulheres militares, através de distintos analisadores da gordura corporal, comparando-os a uma técnica critério, ou gold standard. Portanto, o objetivo desse estudo foi duplo: primeiramente, comparar os valores da gordura corporal relativa informada pelos analisadores Tanita, Omron, Maltron e Futrex com a G\%, via pesagem hidrostática, de mulheres militares do Exército Brasileiro; e, em segundo lugar, determinar a correlação da G\% detectada por cada analisador em relação à G\% obtida via peso hidrostático.

\section{METODOLOGIA}

O presente trabalho caracteriza-se como causal-comparativo e correlacional ${ }^{20}$, pois os valores da $\mathrm{G} \%$, indicados por cada analisador portátil, foram comparados e correlacionados com a G\% critério, obtido pela técnica de pesagem hidrostática.

A amostra foi composta por 30 mulheres militares do Exército Brasileiro (MMEB), residentes na cidade do Rio de Janeiro, com idade entre 20 e 45 anos. Foram excluídas da amostra aquelas que apresentavam uma ou mais das seguintes condições: gravidez, obesidade (IMC $\geq 30 \mathrm{~kg} / \mathrm{m}^{2}$ ), usuárias de diuréticos, portadoras de prótese de silicone, doenças degenerativas ou doenças respiratórias. Essas informações foram obtidas quando da anamnese. O reduzido número de avaliadas é uma limitação do estudo, portanto, os eventuais achados não podem ser generalizados.

O estudo atendeu às normas para a Realização de Pesquisa em Seres Humanos, Resolução 196/96, do Conselho Nacional de Saúde, de 10/10/1996 (sob n 96/04). Todas as participantes assinaram o Termo de Participação Consentida e, duas semanas antes da coleta, foi entregue, para cada voluntária, a relação de cuidados prévios para a análise da bioimpedância corporal, conforme Heyward et al. ${ }^{1}$. 
As mensurações antropométricas da massa corporal (MC, $\mathrm{kg}$ ) e estatura (EST, $\mathrm{cm}$ ) foram efetuadas conforme Norton e Olds ${ }^{21}$. Para a gordura corporal relativa (G\%), usou-se equação de $\mathrm{Siri}^{22}$ e, para a estimativa da G\% pela BIA, os analisadores Maltron BF 900, Tanita UltimateScale 2001, Omron HBF $300{ }^{1,21}$ e o NIR Futrex $5000^{2}$, que antecederam a determinação do peso hidrostático, conforme Behnke et al. ${ }^{3}$, e do volume residual, de acordo com Goldman e Becklake ${ }^{24}$.

A pesagem hidrostática foi efetuada em um tanque específico para tal fim, no laboratório de avaliação do Instituto de Pesquisa e Capacitação Física do Exército (IPCFEx), na cidade do Rio de Janeiro. A descrição das instalações e protocolos foi relatada por Salem et al. ${ }^{25}$.

Estabeleceu-se, também, a validade concorrente (concurrent validity), conforme Morrow Jr et al. ${ }^{26}$, ou seja, a variável critério ( $G \%$ via $P H)$ foi medida quase que simultaneamente à medida estimada (G\% via analisadores).

Com o objetivo de detectar os eventuais erros entre os valores da G\% medida e da G\% estimada, foi calculado o erro constante $(E C$,$) o erro técnico (E T)$, e o erro padrão de estimativa (EPE). Conforme Lohman ${ }^{8}$, os EPE para a G\%, em mulheres, classificam-se em: 2,0\%, Ideal; 2,5\%, Excelente; 3,0\%, Muito Bom; 3,5\%, Bom; 4,0\%, Razoavelmente Bom; 4,5\%, Razoável; e 5,0\%, Não Recomendado.

Os procedimentos matemáticos, para o cálculo dos erros acima indicados, foram os seguintes: para o EC = G\% $\mathrm{PH}$ (critério) - G\% pelo analisador (estimada); $\mathrm{EPE}=\mathrm{s}$ * $\sqrt{1-r^{2}} \quad$ e, o ET $=\sqrt{\sum(G \% p h-G \% e s t)^{2} / n}$

Para a análise estatística, usou-se a descritiva (média e desvio padrão, DP), a correlação simples de Pearson e, na comparação, o teste t pareado de Student entre a G\% critério e a G\% estimada por cada analisador, sendo $\mathrm{p} \leq 0,05$, no SPSS 10,0 .

\section{RESULTADOS}

Os valores descritivos para idade, $\mathrm{MC}(\mathrm{kg}), \mathrm{EST}(\mathrm{cm})$, $\mathrm{D}\left(\mathrm{g} / \mathrm{cm}^{3}\right)$ e $\mathrm{G} \%$ pela $\mathrm{PH}$ das militares estão na TABELA 1 e a $G \%$, estimada através de cada analisador, estão TABELA 2.

Os analisadores Maltron e Tanita, percentualmente, superestimaram a G\%, em relação aos resultados obtidos pela técnica critério, em 0,83 e 9,25\%, respectivamente, enquanto que os analisadores Futrex e Omron subestimaram a G\%, 2,38 e 2,13\%, respectivamente.

A correlação entre a G\% critério, via $\mathrm{PH}$, com a $\mathrm{G} \%$, estimada através de cada analisador, estão relatados na TABELA 3. Todos os valores da G\%, via analisadores, foram significativamente correlacionados, $p<0,001$, com a técnica critério. $\mathrm{O}$ analisador Tanita apresentou a melhor e mais significativa correlação. Os analisadores Maltron, Futrex e Omron apresentaram menores correlações, embora todas significantes, $p<0,001$.

$\mathrm{Na}$ TABELA 3, estão os valores do teste $\mathrm{t}$ e as respectivas diferenças médias entre a G\% critério e a G\% fornecida pelos analisadores. Não houve diferença significante, $\mathrm{p}>0,05$, entre a média da $\mathrm{G} \%$, via $\mathrm{PH}$, com a média da G\% informada pelos analisadores Maltron, Omron e Futrex, exceto para o Tanita, $p<0,05$.

No QUADRO 1, estão as correlações entre técnicas critério e de campo, relatadas por distintos autores, além das características das amostras.

\section{DISCUSSÃO}

Eckerson et al. ${ }^{17}$, Claassen et al. ${ }^{27}$, e Lambert et al. ${ }^{28}$ encontraram correlações altas e significantes, $p<0,05$, $r=0,96,0,85$ e 0,90 , respectivamente, entre absortometria de raios-X de dupla energia (DXA) e Futrex. Entretanto, a correlação entre G\%, via Futrex 5000 e PH, relatado nos estudos de Heyward et al. ${ }^{7}$ e Souza et al. ${ }^{12}$, variou de 0,47 a 0,63 , estando esses valores abaixo do encontrado no presente relato, $r=0,65$, TABELA 3, bem como nos relatos anteriormente citados nesse parágrafo, evidenciando que a homogeneidade das características antropométricas, de magreza, obesidade e sexo, podem influenciar na redução do valor da correlação calculada. Apesar disso, nestes mesmos estudos, não foram encontradas diferenças significantes entre as médias da G\% pelo Futrex e DXA, como também ocorreu no presente estudo, entre o Futrex e $\mathrm{PH}$.

Kushner $^{29}$ recomenda que não é aconselhável estimar a G\% utilizando diretamente os valores fornecidos pelos analisadores de BIA e, sim, as equações específicas e generalizadas desenvolvidas a partir de dados fornecidos pelo analisador como, por exemplo, a resistência, a reatância e/ou a impedância. Como indicado na TABELA 3, não houve diferença entre as médias da G\% fornecida pelo analisador Maltron e a $\mathrm{G} \%$ obtida via $\mathrm{PH}$, tornando 
TABELA 1

VALORES DESCRITIVOS DAS MILITARES.

\begin{tabular}{lccc}
\hline $\mathbf{N}=\mathbf{3 0}$ & $\overline{\mathbf{X}} \pm \mathbf{D P}$ & Mínimo & Máximo \\
\hline Idade, anos & $32,8 \pm 5,7$ & 23,00 & 44,92 \\
$\mathrm{MC}, \mathrm{kg}$ & $57,43 \pm 6,19$ & 48,10 & 72,90 \\
$\mathrm{EST}, \mathrm{cm}$ & $164,8 \pm 5,83$ & 155,00 & 184,20 \\
$\mathrm{D}, \mathrm{g} / \mathrm{cm}^{3}$ & $1,04442 \pm 0,00946$ & 1,02822 & 1,06790 \\
$\% G, \mathrm{PH}$ & $23,98 \pm 4,27$ & 13,52 & 31,41 \\
\hline
\end{tabular}

TABELA 2

VALORES DESCRITIVOS DA G\% POR ANALISADOR.

\begin{tabular}{lccc}
\hline$\% G$ & $\overline{\mathbf{X}} \pm \mathbf{D P}$ & Mínimo & Máximo \\
\hline MALTRON & $24,18 \pm 5,01$ & 15,10 & 38,30 \\
OMRON & $23,47 \pm 4,79$ & 14,10 & 36,80 \\
TANITA & $26,20 \pm 4,93$ & 16,00 & 38,80 \\
FUTREX & $23,41 \pm 5,01$ & 15,20 & 35,20 \\
\hline
\end{tabular}

TABELA 3

VALIDAÇÃO ENTRE G\%, VIA PH (23,98\%G), E G\%, ESTIMADA VIA ANALISADORES, DE MULHERES MILITARES DE 23-44,9 ANOS DE IDADE.

\begin{tabular}{lcccccc}
\hline Analisadores & G\% estimada & $\mathbf{r}^{*}$ & $\mathbf{t}^{* *}$ & $\mathbf{E C}$ & ET & EPE \\
\hline Maltron 900 & 24,18 & 0,69 & $-0,29$ & 0,26 & 0,015 & 3,626 \\
Omron 300 & 23,47 & 0,62 & 0,70 & 0,45 & 0,004 & 3,758 \\
Tanita 2001 & 26,20 & 0,75 & $-3,64^{\star * *}$ & 2,28 & 0,275 & 3,261 \\
Futrex 5000 & 23,41 & 0,65 & 0,78 & 0,51 & 0,130 & 3,807 \\
\hline
\end{tabular}

${ }^{\star} \mathrm{r}=\mathrm{p} \leq 0,001 ;{ }^{* *}$ teste $\mathrm{t}$ de Student; ${ }^{\star \star *} \mathrm{p} \leq 0,01$.

possível o uso desse analisador para as militares do estudo.

Nos estudos de Whatley et al. ${ }^{30}$ e Gibson et al. ${ }^{31}$, que correlacionaram a G\% de mulheres adultas, obtidas via $\mathrm{PH}$ e pelo analisador Tanita, foram encontradas correlações de 0,88 e 0,76 , respectivamente. Esses resultados são superiores ao encontrado no atual estudo $(r=0,75)$, tendo os autores acima informado que houve diferença significativa $(p<0,05)$ entre o critério e o analisador. Contudo, ressalta-se que, apesar da técnica critério ser a mesma, a pesagem hidrostática, os estudos diferem em relação ao modelo do analisador Tanita.
Os achados do presente estudo mostram que a G\%, indicada pelo analisador Tanita, superestimou em 2,2\% a $\mathrm{G} \%$, mensurada via $\mathrm{PH}$, além de ser diferente, $\mathrm{p}<0,05$, do critério. Achados semelhantes foram relatados por Hefferon et al. ${ }^{32}$ (3,09 G\%), Rozo et al. ${ }^{33}$ (entre 2,78 e 3,75 G\%), e Whatley et al. ${ }^{30}$ (em 5,4 G\%), quando analisaram valores de G\% fornecida pelo analisador Tanita. Graham et al. ${ }^{34}$ encontraram uma significante correlação entre Tanita e a Plestimografia $(r=0,92)$, além de uma expressiva subestimação de 4\% na G\% (Tanita = $28 \%$; Plestimografia $=32 \% G$ ). 


\section{QUADRO 1 \\ ESTUDOS COM DIFERENTES ANALISADORES DA GORDURA CORPORAL DE MULHERES.}

\begin{tabular}{|c|c|c|c|c|c|c|c|}
\hline \multirow[b]{2}{*}{ Autor / ano } & \multirow[b]{2}{*}{ Amostra } & \multirow[b]{2}{*}{$\% G$} & \multicolumn{3}{|c|}{ Técnicas } & \multicolumn{2}{|c|}{ Resultados } \\
\hline & & & Critério & Campo & Modelo & $r$ & $\mathbf{p}$ \\
\hline \multirow[t]{2}{*}{ Heyward, et al. ${ }^{7}$} & $\begin{array}{l}\text { Obesas } \\
20 \text { a } 72 \text { anos }\end{array}$ & $>30 \%$ & $\mathrm{PH}$ & NIR Futrex & 5000 & 0,36 & $p<0,05$ \\
\hline & $\begin{array}{l}\text { Não Obesas } \\
20 \text { a } 72 \text { anos }\end{array}$ & $<30 \%$ & & & & 0,58 & $p>0,05$ \\
\hline Eaton, et al. ${ }^{16}$ & $\begin{array}{l}\text { Caucasianas } \\
31,8 \pm 8,6 \text { anos }\end{array}$ & $\begin{array}{c}24,9 \pm 6,6 \% \\
\text { Pesagem Hidrostática }\end{array}$ & $\mathrm{PH}$ & NIR Futrex & ** & 0,47 & $p>0,05$ \\
\hline & & $\begin{array}{c}26,0 \pm 5,5 \% \\
\text { Futrex }\end{array}$ & & & & & \\
\hline Lambert, et al. ${ }^{28}$ & $\begin{array}{l}\text { Adolescentes } \\
12 \text { a } 16 \text { anos }\end{array}$ & * & DXA & NIR Futrex & $6100 \mathrm{~A} / \mathrm{ZL}$ & 0,90 & $p>0,05$ \\
\hline Whatley, et al. ${ }^{30}$ & $\begin{array}{l}\text { Mulheres } \\
20 \text { a } 35 \text { anos }\end{array}$ & $\begin{array}{c}20,8 \pm 2,0 \\
\text { Pesagem Hidrostática }\end{array}$ & $\mathrm{PH}$ & BIA Tanita & TBF 105 & 0,88 & $p<0,05$ \\
\hline & & $\begin{array}{c}26,2 \pm 2,1 \\
\text { Tanita }\end{array}$ & & & & & \\
\hline \multirow[t]{3}{*}{ Rozo, et al. ${ }^{33}$} & 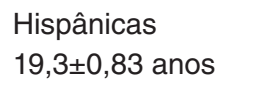 & $\begin{array}{c}13,28 \pm 4,87 \\
\text { Jackson \& Pollock }\end{array}$ & $\mathrm{DC}^{1}$ & BIA Tanita & $2001 \mathrm{~T}$ & 0,78 & $p<0,05$ \\
\hline & & $\begin{array}{c}12,88 \pm 4,59 \\
\text { Kactch \& McArdle }\end{array}$ & & & & 0,81 & $p<0,05$ \\
\hline & & $\begin{array}{c}12,85 \pm 4,58 \\
\text { Yuhasz } \\
16,06 \pm 6,11 \\
\text { Tanita }\end{array}$ & & & & 0,80 & $p<0,05$ \\
\hline Graham, et al. ${ }^{34}$ & $\begin{array}{l}\text { Não atletas } \\
20,9 \pm 2,94 \text { anos }\end{array}$ & $\begin{array}{c}32,0 \pm 8,04 \\
\text { Pletismografia } \\
28,0 \pm 8,94 \\
\text { Tanita }\end{array}$ & PLET $^{2}$ & BIA Tanita & ** & 0,92 & $p<0,05$ \\
\hline Gibson, et al. ${ }^{35}$ & $\begin{array}{l}\text { Mulheres Adultas } \\
18 \text { a } 55 \text { anos }\end{array}$ & $\begin{array}{c}21,8 \\
\text { Pesagem Hidrostática } \\
21,6 \\
\text { Omron }\end{array}$ & $\mathrm{PH}$ & BIA Omron & HBF 300 & 0,83 & $p>0,05$ \\
\hline \multirow[t]{2}{*}{ Gibson, et al. ${ }^{31}$} & Mulheres ativas & $\begin{array}{c}24,8 \pm 7,6 \\
\text { Pesagem Hidrostática }\end{array}$ & $\mathrm{PH}$ & BIA Omron & HBF 300 & 0,87 & $p>0,05$ \\
\hline & $\begin{array}{l}\text { Negras, Hispânicas } \\
\text { e Brancas. } \\
18 \text { a } 59 \text { anos }\end{array}$ & $\begin{array}{c}25,3 \pm 6,8 \\
\text { Omron }\end{array}$ & & & & & \\
\hline \multirow[t]{2}{*}{ Hefferon, et al. ${ }^{32}$} & $\begin{array}{l}\text { Mulheres } \\
18-62 \text { anos }\end{array}$ & $\begin{array}{c}20,28 \pm 0,71 \\
\% G \text { por } \Sigma 7 \text { dobras }\end{array}$ & DC & BIA Omron & HBF 300 & 0,85 & $p>0,05$ \\
\hline & & $\begin{array}{c}20,58 \pm 0,89 \\
\text { Omron }\end{array}$ & & & & & \\
\hline \multirow[t]{2}{*}{ Marques, et al. ${ }^{40}$} & $\begin{array}{l}\text { Brasileiras } \\
20 \text { a } 40 \text { anos }\end{array}$ & $\begin{array}{c}26,25 \pm 5,87 \\
\text { DXA }\end{array}$ & DXA & BIA Omron & HBF 300 & 0,87 & $p<0,05$ \\
\hline & & $\begin{array}{l}21,33 \pm 4,37 \\
\text { Omron }\end{array}$ & & & & & \\
\hline Gartner, et al. ${ }^{38}$ & $\begin{array}{l}\text { Africanas } \\
18 \text { a } 56 \text { anos }\end{array}$ & $\begin{array}{c}34,1 \pm 9,0 \\
\text { Pletismografia } \\
25,3 \pm 8,5 \\
\text { Omron }\end{array}$ & PLET $^{2}$ & BIA Omron & HBF 300 & 0,83 & $p<0,05$ \\
\hline
\end{tabular}

* Não foi indicado; 1 - DC = dobras cutâneas; $2-$ PLET = Pletismografia $\left(\right.$ Bod Pod $\left.{ }^{\circledR}\right)$. 
Conforme os erros constantes, técnicos e de estimativa, mostrados na TABELA 3, observa-se que o analisador Tanita foi o que alcançou os maiores valores relativos nos três tipos de erros, o que pode ser explicado pela significativa diferença $(p<0,05)$ entre as médias da técnica critério e a do analisador, embora este tenha obtido a mais expressiva correlação entre os analisadores. Os valores dos erros constantes e técnicos dos analisadores Maltron, Omron e Futrex foram reduzidos e dentro dos limites, embora ocorram oscilações entre eles. Os EPE dos três analisadores se situaram entre bom e razoavelmente bom e o do Tanita situou-se entre bom e muito bom.

Em distintos relatos, Gibson et al. ${ }^{35}$ correlacionaram os valores da G\%, obtida através do analisador Omron, com a G\%, pela $\mathrm{PH}$, em mulheres com idade entre 18 e 59 anos. Embora a correlação relatada tenha sido significante, o valor da correlação encontrado no presente estudo $(r=0,62)$ foi inferior aos valores relatados por Gibson et al. ${ }^{35}, r=0,87$ e $r=0,83$, respectivamente. Contudo, na comparação, não ocorreram diferenças significantes, $p>0,05$, entre as médias da $\mathrm{G} \%$ via $\mathrm{PH}$ e Omron (0,19 G\%), corroborando com os achados dos dois estudos citados acima, 0,20 G\% e 0,50 G\%, respectivamente. Os relatos de Hefferon et al. ${ }^{32}$, Moreno et al. ${ }^{36}$ e Martin et al. ${ }^{37}$ também informam que não foram encontradas diferenças significantes, $p>0,05$, entre a $G \%$, pelo analisador Omron 300, e a G\%, via equações que utilizam dobras cutâneas.

Contudo, quando o analisador de Omron foi testado via diferentes técnicas critério, como DXA (Martin et al. ${ }^{37}$ ) e Plestimografia (Gartner et al..$^{38}$ e Hayes et al. ${ }^{39}$ ), resultados diferentes dos encontrados nesse estudo foram relatados. Marques et al. ${ }^{40}$, ao testar a validade do analisador Omron 300 , também em mulheres brasileiras com idade entre 20 e 40 anos, concluiu que esse analisador subestimou em $4,92 \%$ a G\%, quando comparada com o critério DXA. Entretanto, deve-se compreender que os valores das variáveis antropométricas, relatados por Martin et al. ${ }^{37}$, Gartner et al. ${ }^{38}$ e Hayes et al. ${ }^{39}$, não são iguais aos analisados no presente artigo. Além disso, Hayes et al. ${ }^{39}$ relatam que foram encontradas diferenças significantes, $p<0,05$, entre as técnicas Omron e Plestimográfica, devido à diferença de modelos dos analisadores Omron utilizados (HBF 300 e HBF 306). Entretanto, Gartner et al. ${ }^{38}$ encontraram uma subestimação ainda maior, de 8,8\%, na G\%, ao usar o analisador Omron. Mas, como a amostra de Gartner et al. ${ }^{38}$ foi constituída por mulheres senegalesas, pode-se, possivelmente, atribuir a grande diferença entre os valores da G\% por conseqüência das diferenças na densidade óssea entre mulheres negras e brancas ${ }^{41}$.

\section{CONCLUSÕES E SUGESTÕES}

Conforme as limitações do presente estudo, concluiu-se que:

- A correlação foi significante entre a G\% critério e as estimadas pelos analisadores Maltron 900, Tanita 2001, Omron 300 e Futrex 5000;

- Os valores da G\%, informados pelos analisadores Maltron 900, Futrex 5000 e Omron 300, não apresentaram diferença significante da G\% critério, obtida via pesagem hidrostática e, por isso, podem ser utilizados para estimar a G\% em mulheres militares do Exército Brasileiro, que apresentem as mesmas características antropométricas e de atividades físicas das analisadas; e

- Os valores da G\% indicados pela balança Tanita 2001, além de serem mais elevados, se diferençaram significativamente da G\% critério.

Sugere-se a realização de mais estudos, com o objetivo de comparar e correlacionar distintas técnicas de análise da composição corporal, em mulheres militares, com um maior número de avaliadas, com características antropométricas diferentes das apresentadas nesse estudo. Ainda, estudos podem ser realizados utilizando as mesmas técnicas (BIA e NIR), mas variando os modelos de equipamentos, bem como repetindo os equipamentos.

\section{REFERÊNCIAS BIBLIOGRÁFICAS}

1. Heyward VH. Evaluation of body composition: current issues. Sports Medicine 1996;22:146-56.

2. Hortobagy T, Israel RG, Houmard JA, McCammon MR, O'Brien KF. Comparison of body composition assessment by hydrodensitometry, skinfolds, and multiple site near-infrared spectrophotometry. European Journal of Clinical Nutrition 1992;3:205-11.

3. Behnke AR, Wilmore JH. Evaluation and regulation of body build and composition. Englewood Cliffs: Prentice-Hall; 1974. 
4. Ellis KJ. Human body composition: in vivo methods. American Physiological Society 2000; 80: 649-80.

5. Oppliger RA, Clark RR, Nielsen DH. New equations improve NIR prediction of body fat among high school wrestlers. $\mathrm{J}$ of Orthopaedic in Sport Physical Therapy 2000; 30: 536-43.

6. Lukaski HC. Methods for assessment of human body composition: traditional and new. American Society for Clinical Nutrition 1987; 46: 537-56.

7. Heyward VH, Cook KL, Hicks VL, Jenkins KA, Quatrochi JA, Wilson WL. Predictive accuracy of three field methods for estimating relative body fatness of nonobese and obese women. International Journal of Sport Nutrition 1992; 2: 75-86.

8. Lohman TG. Advances in body composition assessment. Current issues in exercise science series. Monograph No. 3. Champaign: Human Kinetics, 1992.

9. Carvalho ABR, Pires Neto CS. Desenvolvimento e validação de equações para estimativa da massa corporal magra através da impedância bioelétrica em homens. Revista Brasileira de Atividade Física \& Saúde 1998a; 3:5-12.

10. Carvalho ABR, Pires Neto CS. Desenvolvimento e validação de equações para estimativa da massa corporal magra através da impedância bioelétrica em mulheres. Revista Brasileira de Atividade Física \& Saúde 1998b; 3: 14-21.

11. Lopes AS, Pires Neto CS. Composição corporal em militares utilizando densitometria, antropometria e impedância bioelétrica: um estudo comparativo. Revista Kinesis. 1997;17:15-30.

12. Souza OF, Pires Neto CS, Guimarães FJSP. Comparação e validação cruzada de equações antropométricas e de impedância bioelétrica para estimar a massa corporal magra de alunos do NPOR. Revista Paulista de Educação Física. 1998; 12: 193-201.

13. Neves CEB. Medição da composição corporal por bioimpedância bipolar em resposta a um degrau de tensão. Dissertação de Mestrado. Rio de Janeiro: COPPE; 2000.

14. Rodrigues MN, Silva SC, Monteiro WD, Farinatti PTV. Estimativa da gordura corporal através de equipamentos de bioimpedância, dobras cutâneas e pesagem hidrostática. Revista Brasileira de Medicina do Esporte 2001; 7: 125-31.

15. Cable A, Nieman DC, Austin M, Hogen E, Utter AC. Validity of leg-to-leg bioelectrical impedance measurement in males. Journal Sports Medicine Physical Fitness 2001; 41: 411-4.

16. Eaton AW, Israel RG, O’Brien KF, Hortobagyi T, McCammon MR. Comparison of four methods to assess body composition in women. European Journal of Clinical Nutrition 1993; 47: 353-60.

17. Eckerson J, Moore G, Stout J, Noonan D, Cullen D, et al. Prediction of percent fat using dual-energy x-ray absorptiometry, bioelectrical impedance, and near-infrared interactance. Medicine and Science in Sports and Exercise 1997; 29: Supplement, 53.

18. Smith DB, Johnson GO, Stout JR, Housh TJ, Housh DJ, Evetovich TK. Validity of near-infrared interactance for estimating relative body fat in female high school gymnasts. International Journal Sports Medicine 1997; 18: 531-7.

19. Utter AC, Nieman AN, Ward AN, Butterworth DE. Use of the leg-to-leg bioelectrical impedance method in assessing body composition change in obese women. American Journal of Clinical Nutrition 1999; 69: 603-7.

20. Gay LR. Educational research: Competencies for analysis and application. 3ed. Columbus: Merrill Publishing Company, 1987.

21. Norton K, et al. Técnicas antropométricas. In: Norton K \& Olds T. Antropometrica. Rosário: Biosystem; 2000.

22. Siri WE. Body composition from space and density. In: Brozek J \& Hanschel A. Techniques for Measuring Body Composition. Washington, D. C: National Academy of Science 1961;223-4. 
Revista de Educação Física 2008 Dez; 143:3-11. Rio de Janeiro (RJ) - Brasil.

23. Van Loan MDV. Bioelectrical impedance analysis to determine fat-free mass, total body water and body fat. Sports Medicine 1990; 10: 205-17.

24. Goldman HI, Becklake MR. Respiratory function tests: normal values of medium altitudes and the prediction of normal results. American Review of Tuberculosis and Respiratory Disease. 1959; 79: 457-67.

25. Salem M, Monteiro ABMC, Fernandes Filho J, Pires-Neto CS. Fidedignidade de variáveis antropométricas e da composição corporal pelo peso hidrostático de militares femininas do Exército Brasileiro. Revista Brasileira de Atividade Física e Saúde. 2003;8:45-51.

26. Morrow Jr JM, Jackson AW, Disch JG, Mood DP. Measurement and evaluation in human performance. Champaign: Human Kinetics Books; 1995.

27. Claassen A, Roberts L, Jennings CL, Micklesfield L, Goedecke JH, Dugas L, et al. Modeling \% body fat using near infrared reactance for regularly exercising persons and lean, resistance-trained persons. Medicine and Science in Sports and Exercise 2006; 38: Supplement, S311.

28. Lambert EV, Will M, Micklesfield L, Noakes TD, Lambert MI. Validation of near infrared reactance and impedance techniques for body composition measurement in adolescents. Medicine and Science in Sports and Exercise. 2006; 38: Supplement, S310.

29. Kushner RF. Bioelectrical impedance analysis: A review of principles and applications. Journal of American College of Nutrition 1992;11:205-17.

30. Whatley SW, Florence M, Ransdell LB, Yates JW, Clasey JL. Validity of five bioelectrical impedance analyzers (BIA) used to estimate body composition in young adults. Medicine and Science in Sports and Exercise 1999;31:supplement, S203.

31. Gibson AL, Heyward VH, Jonot JM, Chaves J, et al. Comparison of Tanita and Omron bioimpedance estimates of relative body fatness for physically active women. Medicine and Science in Sports and Exercise 2002; 34: Supplement, S106.

32. Hefferon E, Adamek S, Parks D, Peterson J. Comparison of three consumer grade bioelectrical impedance analyses devices and skinfold measurements. Medicine and Science in Sports and Exercise 2006; 38: Supplement, S313.

33. Rozo D, Lobelo F, Espinosa A, Duperly J. Comparison of skinfold thickness and bioimpedance to assess body composition in young, sedentary, hispanic women. Medicine and Science in Sports and Exercise 2004; 36: Supplement, S71.

34. Graham RE, Nelson JA, Queen BS, Dustin G. A comparison of Air Displacement plethysmography and bioimpedance estimates of body composition in young adults. Medicine and Science in Sports and Exercise 2006; 38: Supplement, S313.

35. Gibson AL, Heyward VH, Mermier CM. Predictive accuracy of Omron® body logic analyzer in estimative relative body fat of adults. Medicine and Science in Sports and Exercise 1999; 31: Supplement, S203.

36. Moreno MV, Gangoy JBG, Gonzales MJA. Medicion de la grasa corporal mediante impedancia bioeletrica, pliegues cutaneos y ecuaciones a partir de medidas antropometricas. Análisis Comparativo. Revista Espanhola de Saúde Pública 2001; 75: 221-36.

37. Martin MV, Gómez GJB, Oya OM, Gómez CA, Antoranz GM. Grado de acuerdo entre los índices adiposo-musculares obtenidos a partir de medidas antropométricas del brazo, pliegues cutáneos e impedancia bioelétrica. Nutricion Hospitalaria 2003;18:77-86.

38. Gartner A, Dioum A, Delpeuch F, Maire B, Schutz Y. Use of hand-to-hand impedancemetry to predict body composition of african woman as measured by air plethysmography. European Journal of Clinical Nutrition 2004; 58: 523-31. 
39. Hayes HM, Kern M. Comparison of air displacement plethysmography with the Omron body fat analyzer. Medicine and Science in Sports and Exercise 2006; 38: Supplement, S309-S310.

40. Marques MB, Heyward VH, Fa CSM, Mermier CM. Validação cruzada de equações de BIA em mulheres brasileiras por meio de DEXA. Revista Brasileira de Ciência e Movimento. 2000;8:14-20.

41. Adams WC, Deck-Coté K, Winters KM. Anthropometric estimation of bone mineral content in young adult females. American J Human Biology 1992; 4:767-74.

\section{Endereço para correspondência:}

Avenida Sernambetiba, 4000, bl. 05, apt. 106

Barra da Tijuca - RJ - Brasil

CEP: 22630-011

e-mail: anabeatrizmcm @yahoo.com.br

\section{PUBLIQUe seUS ARTIGOS NA REVISTA de EDUCAÇÃO FísICa}

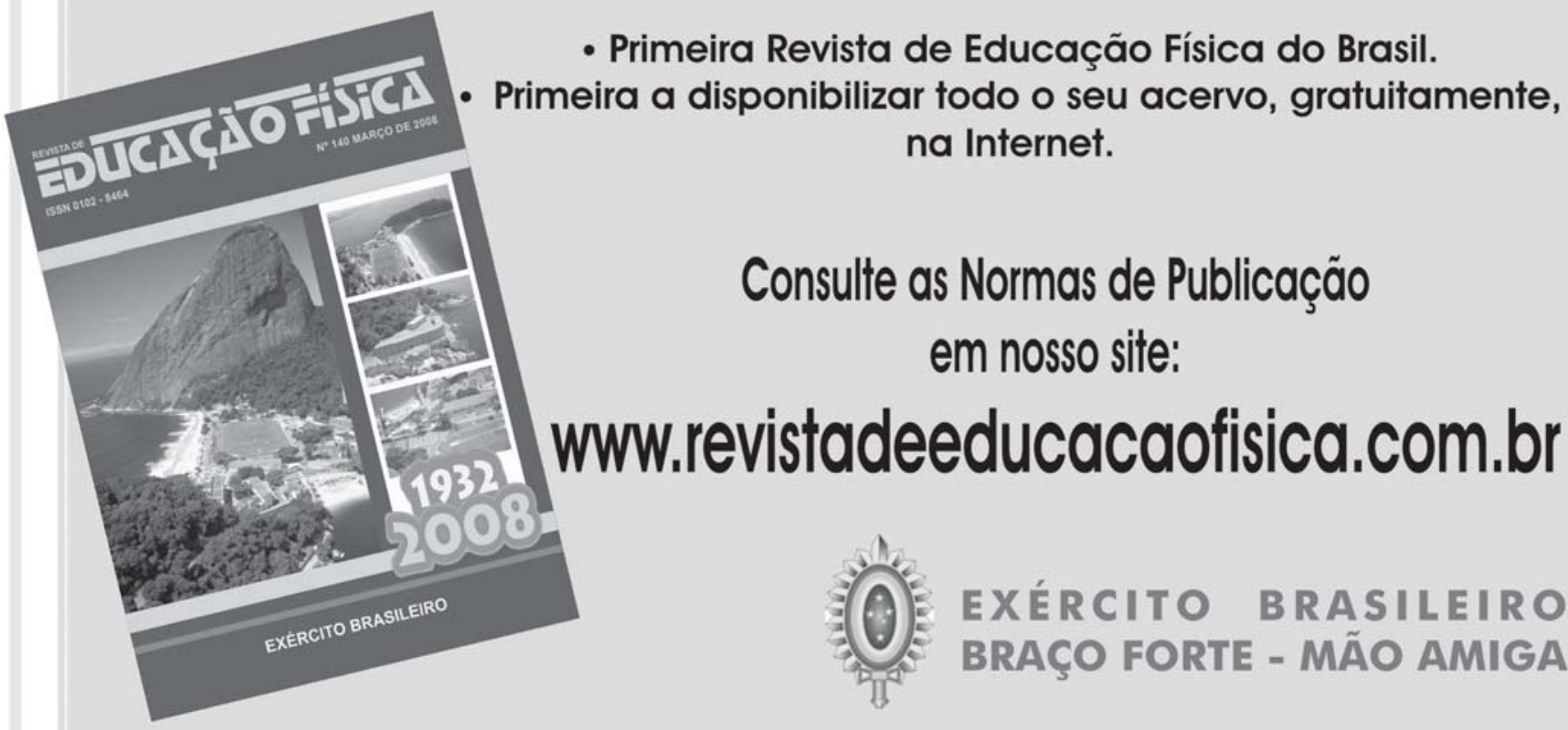

